\title{
Using X-ray Flux Time Series for Solar Explosion Forecastings
}

\author{
Ismael Caldana*, Ana Estela Antunes da Silva
}

\begin{abstract}
Among the natural phenomena that happen in the Sun, one that has direct impact on Earth are the solar explosions. These explosions emit an X-ray flux that can be detected and used as an indicative of new explosions. In this work, we evaluated whether X-ray flux time series are suitable as the base dataset for solar flare forecasting. To do so, we applied a multilayer perceptron (MLP) neural network. The experiments indicated that X-ray flux time series can be used to forecast solar flares, even though the average errors increase with wider forecast horizons.
\end{abstract}

\section{Key words:}

neural network, space weather, forecasting.

\section{Introduction}

During solar cycles, which occur at eleven-year intervals, the Sun exhibits characteristic behaviors called solar activity, wherein the solar explosions are one of these phenomena [1]. The X-ray flux emitted by the sun is one of the factors used to classify the explosions. The flow, measured in Watts per square meter $\left(\mathrm{W} / \mathrm{m}^{2}\right)$, is used on a logarithmic scale that divides the eruptions into the categories: $A, B, C, M$ and $X$ (from lowest to highest intensity) [2].

The main aim of this study was to establish the possibility of using $x$-ray flux as a predictive parameter of solar flares. A Multilayer Perceptron (MLP) neural network was applied to the X-ray flux time series obtained from the GOES-15 satellite from January 1, 2014 to December 31, 2014 [3]-[4].

\section{Results and Discussion}

Several experiments were conducted and the results showed that such prediction is possible if short forecasting horizons are considered. The summary of the last used MLP topology is:

- Input Layer: 05 neurons with logistic activation functions; the inputted values correspond to 05 consecutive historical values of the time series immediately before the first value to be forecast by the MLP;

- Hidden Layer: 10 neurons with logistic activation functions;

- Output Layer: 1 neuron with logistic activation function.

The dataset used in the experiments were submitted to a balancing process and five training algorithms was evaluated. Quick Propagation was chosen because it leaded to the smallest X-ray flux forecast errors for different forecast horizons. The Table 1 shows the obtained values.

Table 1. Presentation of the average of the Mean Squared Error of Prediction for different forecast horizons

\begin{tabular}{|c|c|c|}
\hline One minute & Five minutes & Ten minutes \\
\hline $7,49 * 10^{-14}$ & $5,21 * 10^{-12}$ & $3,04 * 10^{-11}$ \\
\hline
\end{tabular}

The Figure 1 represents of a comparison between the values obtained by the neural network and the ideal values.

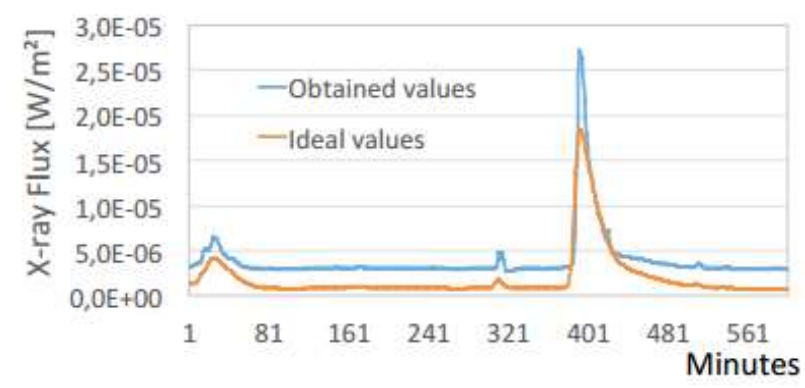

Figure 1. Cut of the original and forecast series, for a five minutes forecast horizon, obtained by an MLP.

\section{Conclusions}

In this work, we have evaluated whether X-ray flux time series is suitable to be used to forecast solar explosions of different intensities and in different forecast horizons. In summary, the experimental results indicated that even though the errors increase with the extension of the forecast horizon, MLPs with a single output and specialized in predicting future values of a particular horizon led to the smallest mean-squared errors. As future works, several extensions that can be derived from this work. First, a larger time series of X-ray flux should be considered. Second, different machine learning approaches could also be adopted. Finally, wider forecast horizons could be explored.

\section{Acknowledgement}

I would like to thank my advisor Ana Estela, the UNICAMP and also FAPESP for making this research possible.

\footnotetext{
1 R. Schwenn, "Space Weather: The Solar Perspective," Living Reviews in Solar Physics, vol. 3, no. 1, p. 2, 2006.

2 K. C. Fox, "Classifying Solar Eruptions," 2012. [Online]. Available: https://www.nasa.gov/mission_pages/sunearth/news/classify-flares.html

3 S. W. P. Center, "NWS Space Weather Prediction Center," 2015. [Online]. Available: http://www.swpc.noaa.gov

4 Han, M. Kamber, and J. Pei, Data Mining: Concepts and Techniques, 3rd ed. Illinois: Morgan Kaufmann Publishers, 2006.
} 